\title{
Kinetics of amorphization induced by swift heavy ions in $\alpha$-quartz
}

\author{
O. Peña-Rodríguez \\ J. Manzano-Santamaría \\ A. Rivera \\ G. García J. Olivares \\ F. Agulló-López
}

\begin{abstract}
A B S T R A C T
The kinetics of amorphization in crystalline $\mathrm{SiO}_{2}\left(\alpha\right.$-quartz) under irradiation with swift heavy ions $\left(\mathrm{O}^{+1}\right.$ at $4 \mathrm{MeV}, \mathrm{O}^{+4}$ at $13 \mathrm{MeV}, \mathrm{F}^{+2}$ at $5 \mathrm{MeV}, \mathrm{F}^{+4}$ at $15 \mathrm{MeV}, \mathrm{Cl}^{+3}$ at $10 \mathrm{MeV}, \mathrm{Cl}^{+4}$ at $20 \mathrm{MeV}, \mathrm{Br}^{+5}$ at 15 and $25 \mathrm{MeV}$ and $\mathrm{Br}^{+8}$ at $40 \mathrm{MeV}$ ) has been analyzed in this work with an Avrami-type law and also with a recently developed cumulative approach (track-overlap model). This latter model assumes a track morphology consisting of an amorphous core (area $\sigma$ ) and a surrounding defective halo (area $h$ ), both being axially symmetric. The parameters of the two approaches which provide the best fit to the experimental data have been obtained as a function of the electronic stopping power $S_{e}$. The extrapolation of the $\sigma\left(S_{e}\right)$ dependence yields a threshold value for amorphization, $S_{t h} \approx 2.1 \mathrm{keV} / \mathrm{nm}$; a second threshold is also observed around $4.1 \mathrm{keV} / \mathrm{nm}$. We believe that this double-threshold effect could be related to the appearance of discontinuous tracks in the region between 2.1 and $4.1 \mathrm{keV} / \mathrm{nm}$. For stopping power values around or below the lower threshold, where the ratio $h / \sigma$ is large, the track-overlap model provides a much better fit than the Avrami function. Therefore, the data show that a right modeling of the amorphization kinetics needs to take into account the contribution of the defective track halo. Finally, a short comparative discussion with the kinetic laws obtained for elastic collision damage is given.
\end{abstract}

\section{Introduction}

The structural modifications induced by charged (or neutral) particles on materials [1-3] are a key issue to allow for the reliable operation of nuclear fission reactors and to minimize the deleterious effects associated to the storage of the nuclear radioactive waste $[4,5]$. The damage problem may be even more demanding for the future fusion reactors given the high neutron fluxes and temperatures reached during operation of the confined plasma [6] or the extreme power of laser pulses for inertial systems [7]. Moreover, there are many other technologies where ion-beam damage is, or could be, quite relevant such as ion-implantation, ion-beam patterning, lifetime of components and devices in space missions and hadron therapy.

The amorphization of crystalline materials by ion beams is usually produced during the final stages of the damage, when the degree of lattice disorder has reached a sufficiently large value. Depending on the kind and energy of the incident ion, the damage for dielectric materials can be predominantly produced either by elastic collisions between the bombarding particles and the atoms of the material (i.e., the damage related to the nuclear stopping power, $S_{n}$ ) or by the relaxation of the electronic excitations produced by the incoming particles (i.e., the damage associated with the electronic stopping power, $S_{e}$ ). Out of these two contributions, the nuclear damage and amorphization has been extensively studied [8-11], whereas damage in the electronic stopping regime is still poorly understood. However, it is becoming increasingly clear that the relaxation of the high electronic excitation associated with $S_{e}$ plays a fundamental role in the ion-beam induced damage and the subsequent amorphization process [12,13], at least in the case of dielectric materials. It has been widely documented that the impacts of swift heavy ions (where the electronic stopping regime dominates) on several dielectric targets generate individual tracks along the trajectory, whenever a certain threshold stopping power is overcome [14-16]. These observations are often explained through a thermal spike approach involving lattice melting and re-solidification of the heated material around the ion trajectory $[17,18]$. More recently, a different theoretical scheme has been developed for some crystals (e.g., $\mathrm{LiNbO}_{3}$ ) in which the damage process, including track formation, is analyzed as a cumulative process that occurs via formation of point defects. When the defect concentration reaches a certain threshold value the lattice collapses into an amorphous phase. Several specific models for defect creation have been proposed, including thermally-assisted bond 
breaking [19] and non-radiative exciton decay [20,21]. We consider that these models appear better suited to account for the structure and morphology of the damage tracks consisting of an amorphous core surrounded by a halo of point (and maybe extended) defects. Unfortunately, direct experimental evidence for the occurrence and structure of the halo is scarce [22-25] due to the lack of enough structural sensitivity of TEM, and RBS/C but there have been some new developments; for instance, SAXS has been recently used to study the morphology of the tracks in silica [26]. Anyhow, the amorphization kinetics offers a relevant method to monitor the evolution and structure of ion-beam damage as illustrated in this paper.

As for the amorphization kinetics in the electronic excitation regime, it is generally accepted that for low-enough stopping powers it is sigmoidal (Avrami-type), with a null or very small initial slope [27-31]. When the stopping powers are increased above the threshold value for track generation, then the Avrami kinetics turns into a Poisson behavior. All those data suggest that the damage proceeds through accumulation of radiation-induced defects that finally collapse into a new amorphous phase. Therefore, one expects that the kinetics of accumulation provides relevant clues to understand the structure of individual tracks. We have recently proposed a general cumulative scheme to describe damage [32], which is independent of the particular mechanism causing bond breaking and defect formation. It assumes that every impact generates a track consisting of an amorphous core of cross-section $\sigma$ surrounded by a halo of area $h$. Moreover, we assumed that the superimposition of a fixed number $N$ of halos results in amorphization. The model was applied to fit some data for $\mathrm{LiNbO}_{3}$ [32] and the results were very stimulating. In addition, a Monte Carlo code based on such specific defect-creation model for $\mathrm{LiNbO}_{3}$ has been able to simulate the morphology and kinetics of amorphization [33]. Finally, it is important to mention that although in the previous discussion we have implied that the pristine material is crystalline and the damaged track is amorphous this is not a requirement of our model but a limitation of data from the RBS/C technique. If the damage kinetics is obtained by a different method (e.g., optical techniques [34]), able to distinguish between the properties of damaged and pristine regions, then, the track-overlap approach remains perfectly applicable, even in the absence of amorphization.

The purpose of this paper is to discuss the kinetic data obtained by swift-ion irradiation on crystalline $\mathrm{SiO}_{2}$ ( $\alpha$-quartz), covering a wide range of electronic stopping powers around and above the threshold value. Although some rather similar studies have been reported $[18,27]$, they are based on the concept of thermal spike and, consequently, they do not analyze the generation and accumulation of point defects that we consider a key point for a proper understanding of the amorphization process. Our experimental curves have been simulated with an Avrami-type kinetics as well as by the recently proposed track-overlap (TO) model [32]. For this latter case, the physical parameters $(\sigma, h$ and $N)$ have been determined as a function of the electronic stopping power $S_{e}$. Aside from providing a more physical insight into the damage kinetics; this model appears better suited to describe the experimental data than the Avrami curves, particularly in the initial low-fluence region (i.e., where the accumulated damage is still low). Some implications of these results on the structure and mechanisms of electronic damage are discussed.

\section{Track-overlap model}

Here, we summarize the main conclusions and formulae for the recently proposed track-overlap model [32]. In this approach we have assumed that every impact generates a cylindrical track con- sisting of an inner amorphous core of cross-section $\sigma$, surrounded by a homogeneous defective halo of area $h$ in the transversal plane (Fig. 1). The superimposition of a fixed number $N$ of halos leads to local amorphization, whereas the amorphous structure is not further modified by the track overlap. This model resembles the approach developed by Gibbons [35] to describe amorphization by elastic nuclear collisions from light ions, as well as the pre-damage effect. It assumes that every projectile ion creates a heavily damaged region through atomic displacements (defect cluster) that becomes amorphous after $\mathrm{N}$-tuple overlapping. However, our model is more general, as long as it includes a more complex morphology of the damage tracks and the overlapping between amorphous and pre-amorphous zones. Moreover, the structure of damage by swift ions appears better defined and has sharper amorphous-crystalline boundaries, which is more suitable for the model. Then, the general formula giving the total amorphous area $S$ as a function of impact density $\phi$ (fluence) is [32]:

$S(\phi)=1-\exp (-\sigma \phi) \exp (-h \phi)\left(1+\sum_{i=1}^{N-1} h^{i} \phi^{i} / i !\right)$

where $N$ is the number of successive impacts required to amorphize the crystal by overlapping of halos. One important feature of that expression is that the halo also contributes to the initial slope of the curve and so the kinetics is not strictly Poisson even for $\sigma \neq 0$ (i.e., when every ion impact produces direct amorphization). Of course, if $h \ll \sigma$ (predominance of the core area) the Poisson kinetics is, very approximately, recovered. Another relevant feature of Eq. (1) is that, for $\sigma=0$, the initial region of the curve grows as $h^{N}$.

Now, the experimental data can be adjusted using expression (1) and the following procedure. Firstly, a linear fit is done for the low-fluence data, in order to derive an approximation for the value of the parameter $\sigma$. If the $y$-intercept of the fitted line yields a value below zero (statistically significant), then the result is interpreted as $\sigma=0$. If the fit is compatible with the fitted line crossing the origin, then $\sigma$ is just its slope. Then, the value of $h$ is determined from a fit, performed with $\sigma$ fixed to the previously

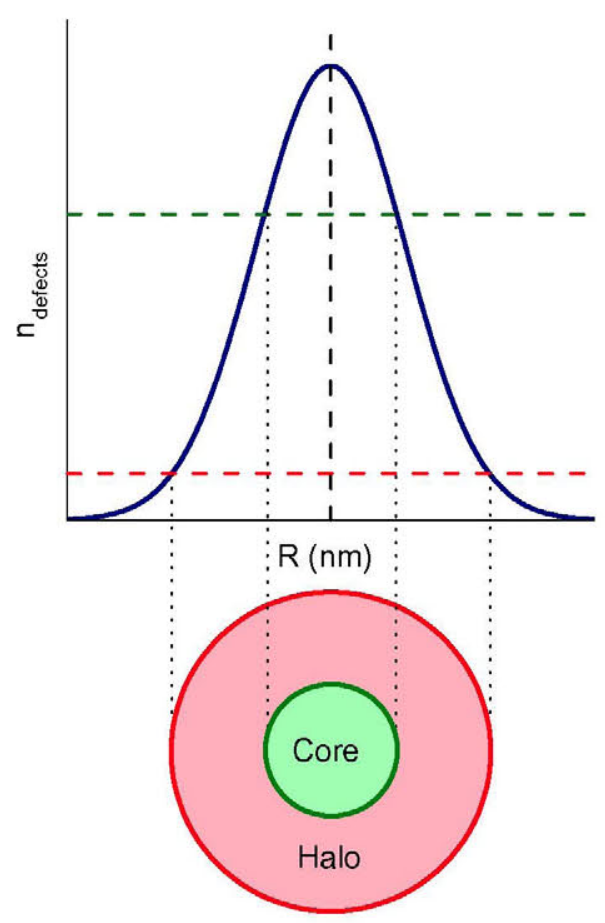

Fig. 1. Schematic representation of the ion tracks used in the track-overlap model, they consist of an amorphous core surrounded by a partially-damaged halo. 
Table 1

Electronic stopping power and projected range for the ions used in this work, calculated using SRIM $2008[38,39]$.

\begin{tabular}{llll}
\hline lon & Energy $(\mathrm{MeV})$ & $S_{e}(\mathrm{keV} / \mathrm{nm})$ & $R_{p}(\mu \mathrm{m})$ \\
\hline Oxygen & 13 & 2.0 & 7.3 \\
Oxygen & 4 & 2.1 & 3.0 \\
Fluorine & 15 & 2.3 & 7.3 \\
Fluorine & 5 & 2.5 & 3.3 \\
Chlorine & 10 & 4.4 & 3.9 \\
Chlorine & 20 & 5.0 & 6.0 \\
Bromine & 15 & 5.4 & 4.8 \\
Bromine & 25 & 6.9 & 6.4 \\
Bromine & 40 & 8.3 & 8.3 \\
\hline
\end{tabular}

obtained value. Finally, a second fit is done with both parameters free. This procedure is identical to the one we used in the original paper on the track-overlap model [32].

\section{Experimental}

Single crystal synthetic quartz samples, $1 \mathrm{~mm}$ thick and with a surface area of $7 \times 8 \mathrm{~mm}^{2}$, were used for these experiments. They were irradiated in the $5 \mathrm{MV}$ Tandetron accelerator at Centro de Micro-Análisis de Materiales (CMAM) [36], using an angle of five degrees with respect to the [0001] sample normal to avoid the channeling condition. Different energies and ions were selected, in order to vary the electronic stopping power from slightly below to far above the amorphization threshold, estimated to be $\sim 2 \mathrm{keV} /$ $\mathrm{nm}$ [37]. The irradiations were performed using $\mathrm{O}^{+1}$ at $4 \mathrm{MeV}, \mathrm{O}^{+4}$ at $13 \mathrm{MeV}, \mathrm{F}^{+2}$ at $5 \mathrm{MeV}, \mathrm{F}^{+4}$ at $15 \mathrm{MeV}, \mathrm{Cl}^{+3}$ at $10 \mathrm{MeV}, \mathrm{Cl}^{+4}$ at $20 \mathrm{MeV}, \mathrm{Br}^{+5}$ at 15 and $25 \mathrm{MeV}$ and $\mathrm{Br}^{+8}$ at $40 \mathrm{MeV}$; with fluences ranging from $2 \times 10^{12} \mathrm{~cm}^{-2}$, where almost no amorphization is produced, to $2 \times 10^{14} \mathrm{~cm}^{-2}$, where the sample surface was completely amorphized. In all cases the ion current was kept below $20 \mathrm{nA}$ (ion flux between $10^{9}$ and $10^{10}$ particles/( $\mathrm{cm}^{2}$.'s) to avoid sample heating. The electronic stopping powers at the sample surface and the projected range, calculated using SRIM 2008 [38,39], are given in Table 1 . They show that the electronic stopping regime is clearly dominant. Small metallic masks were fixed to the surface of the samples, in order to avoid electrical discharges.

The disorder induced by the irradiation was measured by RBS channeling (RBS/C), with helium at $3.035 \mathrm{MeV}[29,40]$. Some typical RBS/C spectra, obtained from the samples irradiated with $\mathrm{Cl}^{+3}$ at $10 \mathrm{MeV}$, are illustrated in Fig. 2. The induced amorphous (disor-

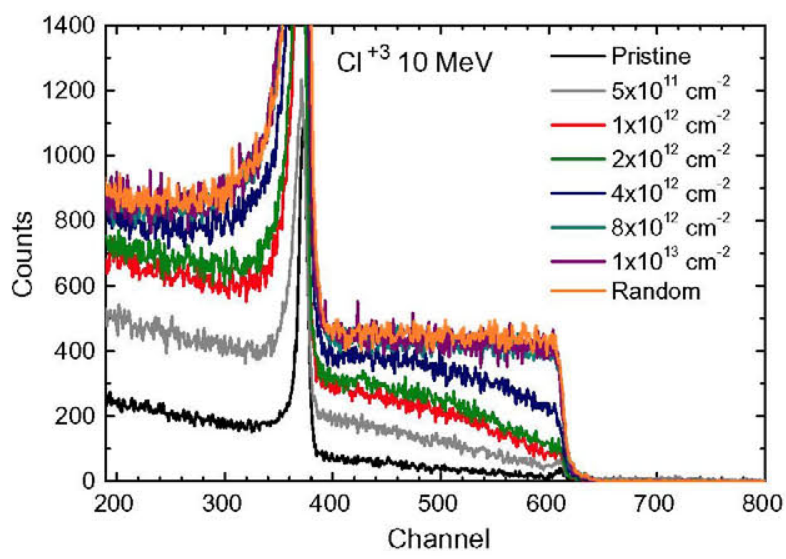

Fig. 2. Representative RBS/C spectra measured with a probe ion beam of He at $3.035 \mathrm{MeV}$, corresponding to the irradiations with $\mathrm{Cl}^{+3}$ at $10 \mathrm{MeV}$. The irradiation fluences are indicated in the figure. dered) fraction $f$ at the surface of the sample is obtained through the standard expression for crystalline materials [40],

$f=\frac{\chi_{i}(\text { channeled })-\chi_{v}(\text { channeled })}{\chi_{v}(\text { random })-\chi_{v}(\text { channeled })}$

where $\chi$ is the RBS yield, either in channeled or random configuration, and the subindices $v$ and $i$ denote the virgin (i.e., pristine) and irradiated samples, respectively.

\section{Results and discussion}

Fig. 3 shows the experimental curves, depicting the disordered atomic fraction at the surface of a quartz sample as a function of the irradiation fluence for the selected ions. The damage curves span a wide range of fluences, since we obtain the area of the core and the halo from the fit of the whole kinetics. In other words, samples must be irradiated until complete surface amorphization is obtained and this value is dependent upon the ion and energy used. Independent measurements of the threshold for track amorphization yield $S_{t h} \approx 2 \mathrm{keV} / \mathrm{nm}$ [37]; based on this, we selected the analyzed cases trying to cover the region going from below to far above such threshold. The experimental curves clearly show the expected trend; i.e., sigmoidal kinetics for low stopping powers that progressively changes to Poisson behavior above the threshold.

\subsection{Data analysis with an Avrami kinetics}

The common practice in the literature is to fit these experimental curves using the Avrami kinetics, given by the expression:

$f=1-\exp \left\{-\left(\phi / \phi_{0}\right)^{n}\right\}$

where the two parameters, $n$ and $\phi_{0}$, account for the abruptness of the rising part of the sigmoid and for the initial incubation fluence, respectively. The Avrami kinetics offers a reasonable overall description of experimental curves for the lighter ions, as shown in Fig. 3. However, it cannot accurately describe the initial stage of amorphization for stopping powers near or slightly above threshold, as illustrated in the same figure. This is because the initial slope of any Avrami kinetics (with $n>1$ ) is zero, whereas experimental curves show a finite initial slope. In other words, Avrami's parameters are not sensitive enough to distinguish between the subthreshold and above-threshold regimes. The discrepancy may be a consequence of the complex morphology of the damage tracks, demanding for a more physical model that explicitly includes the

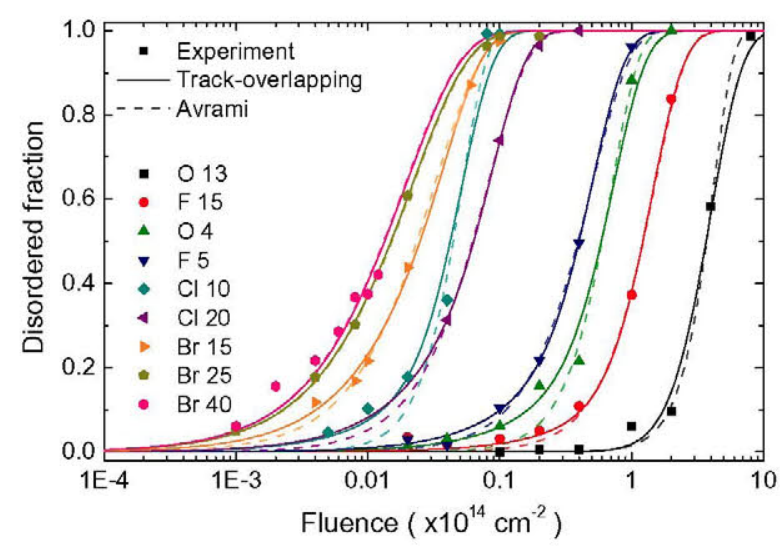

Fig. 3. Fit of damage fraction curves with track-overlap model (continuous lines) and Avrami kinetics (dashed lines). 


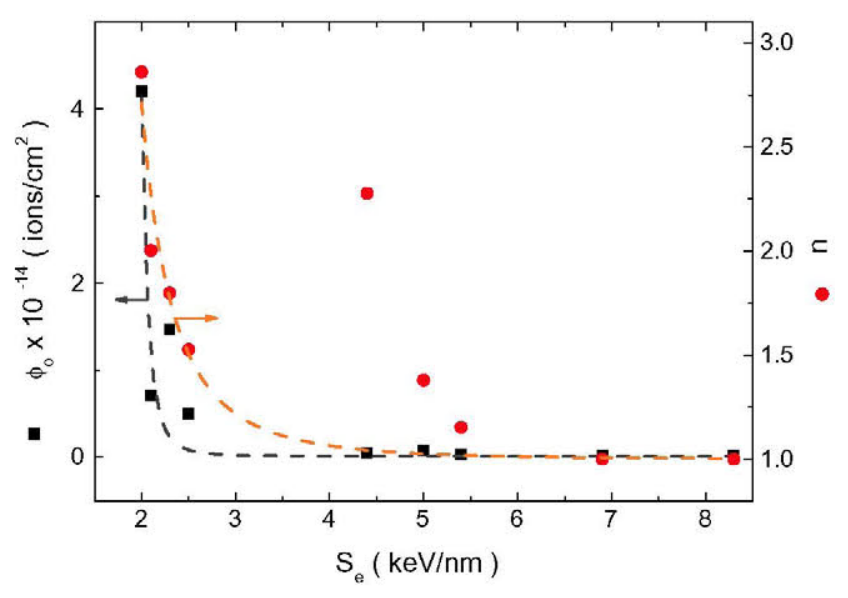

Fig. 4. Dependence of $\phi_{0}$ and $n$ on stopping power. The lines are just a guide to the eye.

Table 2

Values of the Avrami model parameters $\phi_{0}$ and $n$, obtained from the fits.

\begin{tabular}{lrll}
\hline Ion & Energy $(\mathrm{MeV})$ & $n$ & $\phi_{0}\left(\mathrm{~cm}^{-2}\right)$ \\
\hline Oxygen & 13 & $2.9 \pm 0.3$ & $(4.2 \pm 0.5) \times 10^{14}$ \\
Oxygen & 4 & $2.1 \pm 0.2$ & $(7.1 \pm 0.8) \times 10^{13}$ \\
Fluorine & 15 & $1.8 \pm 0.1$ & $(1.5 \pm 0.3) \times 10^{14}$ \\
Fluorine & 5 & $1.5 \pm 0.1$ & $(5.0 \pm 0.5) \times 10^{13}$ \\
Chlorine & 10 & $2.3 \pm 0.2$ & $(5.2 \pm 0.5) \times 10^{12}$ \\
Chlorine & 20 & $1.4 \pm 0.1$ & $(8.1 \pm 0.7) \times 10^{12}$ \\
Bromine & 15 & $1.2 \pm 0.1$ & $(3.2 \pm 0.4) \times 10^{12}$ \\
Bromine & 25 & $1.0 \pm 0.1$ & $(2.2 \pm 0.2) \times 10^{12}$ \\
Bromine & 40 & $1.0 \pm 0.1$ & $(1.9 \pm 0.2) \times 10^{12}$ \\
\hline
\end{tabular}

inner amorphous core and the surrounding defective halo (trackoverlap model).

In Fig. 4 we plot the dependence of the Avrami parameters on stopping power $S_{e}$; the model's parameters obtained from the fit are also shown in Table 2. The dependence is in accordance with that obtained for other dielectric materials [28-30] and confirms a general trend: $n \gg 1$ for stopping powers below threshold and decreases down to an asymptotic value, $n=1$, for high enough stopping powers $\left(S_{e} \gg S_{t h}\right)$ [30]. In other words, one should remark that the exponent $n$ remains above 1 even for stopping powers somewhat higher than the threshold value. Therefore, this result poses some caution on the usual procedure of determining the threshold stopping power values $S_{t h}$ by extrapolation of the initial slope of the amorphization curve.

\subsection{Analysis by the track-overlap model}

For comparison purposes we have also fitted all the experimental data in Fig. 3 with Eq. (1) for the track-overlap model (continuous lines) using appropriate parameters $(N, \sigma$ and $h)$, which are physically meaningful. The optimal value of $N$ was determined by fitting the data with the expressions obtained for several values of $N$, ranging from 2 to 7 (not shown). It was found that a value $N=4$, precisely the same value that we had previously obtained for lithium niobate [32], provided the best fits [41]. On the other hand, one sees that the TO model also provides a good quantitative description of the experimental data, like its Avrami counterpart, but appears better suited to describe the experimental points in the low-fluence region. The contribution of the halo, as determined from this model, to the damage kinetics is illustrated in Fig. 5 for the case of $\mathrm{F}$ at $5 \mathrm{MeV}$ and $\mathrm{Br}$ at $15 \mathrm{MeV}$.
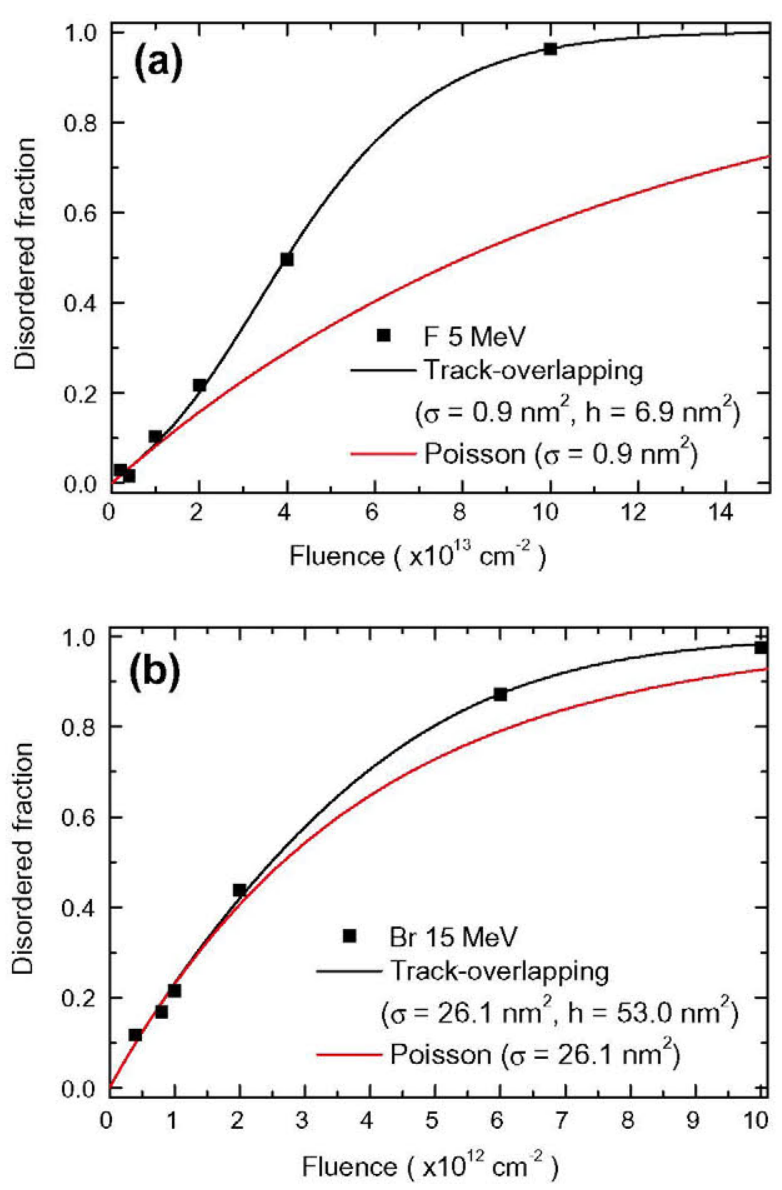

Fig. 5. Decomposition of the contribution of the core (Poisson) and the halo (difference between the total curve and the Poisson one) in the TO approach for fluorine at $5 \mathrm{MeV}$ (a) and bromine at $15 \mathrm{MeV}$ (b).

Table 3

Values of the track-overlap model parameters $\sigma$ and h, obtained from the fits and radius of the core $\left(r_{c}\right)$ and thickness of the halo $\left(t_{h}\right)$, calculated from the areas by assuming a cylindrical track.

\begin{tabular}{lcllll}
\hline Ion & Energy $(\mathrm{MeV})$ & $\sigma\left(\mathrm{nm}^{2}\right)$ & $h\left(\mathrm{~nm}^{2}\right)$ & $r_{c}(\mathrm{~nm})$ & $t_{h}(\mathrm{~nm})$ \\
\hline Oxygen & 13 & 0 & $1.0 \pm 0.1$ & 0 & 0.6 \\
Oxygen & 4 & $0.6 \pm 0.2$ & $4.6 \pm 0.8$ & 0.5 & 0.8 \\
Fluorine & 15 & $0.2 \pm 0.1$ & $2.5 \pm 0.5$ & 0.3 & 0.7 \\
Fluorine & 5 & $1.0 \pm 0.5$ & $6.4 \pm 0.9$ & 0.6 & 1.0 \\
Chlorine & 10 & $7 \pm 1$ & $68 \pm 10$ & 1.5 & 3.4 \\
Chlorine & 20 & $9 \pm 1$ & $31 \pm 5$ & 1.6 & 1.9 \\
Bromine & 15 & $26 \pm 4$ & $53 \pm 8$ & 2.9 & 2.1 \\
Bromine & 25 & $46 \pm 7$ & 0 & 3.8 & 0 \\
Bromine & 40 & $52 \pm 9$ & 0 & 4.1 & 0 \\
\hline
\end{tabular}

The best-fit values for the TO model parameters, $\sigma$ and $h$, are listed in Table 3 and plotted as a function of stopping power $S_{e}$ near the threshold value in Fig. 6 . We can see that for oxygen and fluorine the core areas $\sigma$ are comparatively small $\left(<1 \mathrm{~nm}^{2}\right)$ and the track morphology is strongly dominated by the halo (a few square nanometers). This is due to the close proximity of $S_{e}$ to the threshold value; indeed, $\sigma=0$ for irradiation with $\mathrm{O}$ at $13 \mathrm{MeV}\left(S_{e}=2.0\right.$ $\mathrm{keV} / \mathrm{nm}$ ) where we are just below the threshold. The dependence of $\sigma$ on stopping power is approximately logarithmic for the region $2.0 \mathrm{keV} / \mathrm{nm}<S_{e}<5.0 \mathrm{keV} / \mathrm{nm}$, allowing for a precise determination of the threshold $(2.1 \mathrm{keV} / \mathrm{nm})$ using the equation developed by Szenes $\left(R^{2}=A \ln \left(S_{e} / S_{e}^{\text {th }}\right) ;\right.$ A being a proportionality constant and 


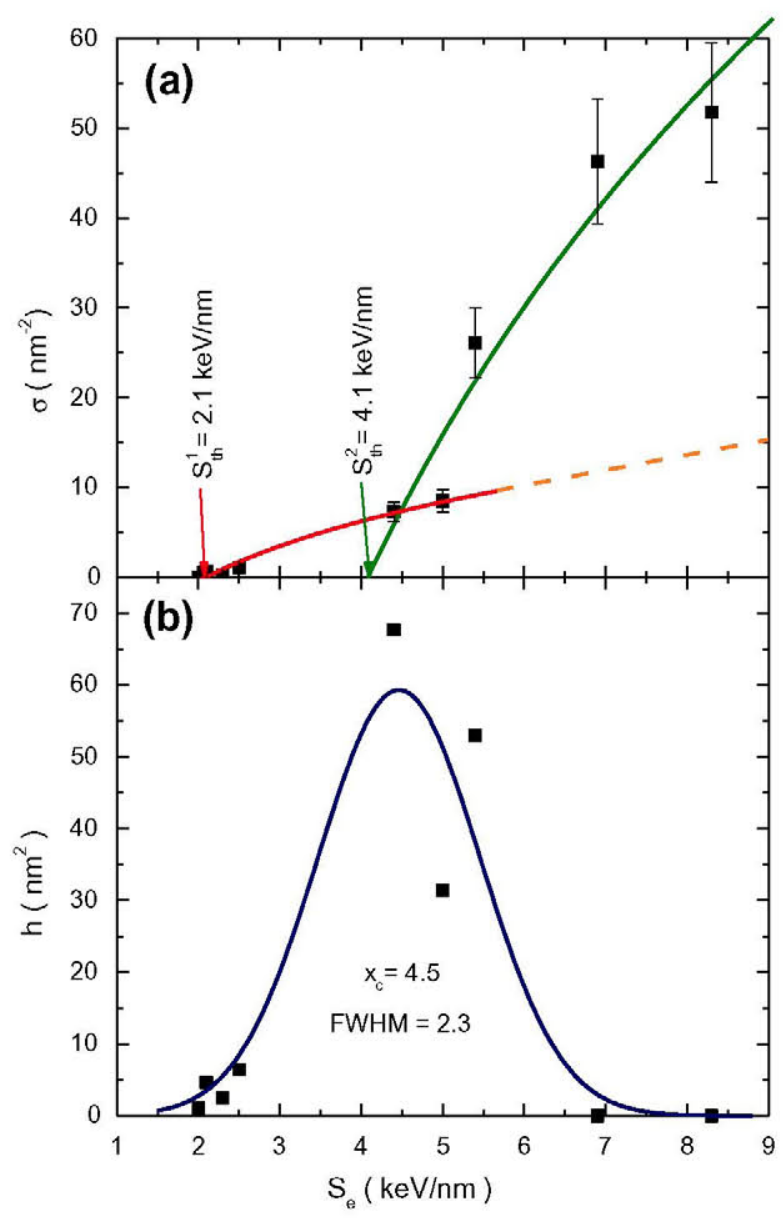

Fig. 6. Dependence of $\sigma$ (a) and $h$ (b) on stopping power. The continuous lines in (a) are a fit of the data using the model developed by Szenes [42] for the slow (red) and fast (green) grow regions, whereas the orange dashed line represents the lineargrowth region. The blue continuous line in (b) is a Gaussian fit of the h values.

$S_{e}^{\text {th }}$ the threshold stopping power) [42] (Fig. 6a). This initial slowgrow region is followed by a rapidly growing stage, which finally slows down. It should be noted that the increase observed in the second region is far higher than the one expected for the linear zone above $S_{e} / S_{t h}>2.7$ [42] (orange ${ }^{1}$ dashed line); a similar effect has been also observed in $\mathrm{LiNbO}_{3}$ [43]. A new extrapolation with Szenes' model of the rapidly increasing stage suggests the existence of a second threshold at around $4.1 \mathrm{keV} / \mathrm{nm}$ (Fig. 6a). It is not clear to us which is the origin of this double threshold but one can speculate that the region between both thresholds may correspond to discontinuous track cores; this effect has been already reported for a number of different materials $[18,44,45]$.

On the other hand, the curve, $h$ vs. $S_{e}$ (Fig. 6b), grows steadily up to a maximum around $4 \mathrm{keV} / \mathrm{nm}$ and then, decreases down to zero. Up to $S_{e}=5 \mathrm{keV} / \mathrm{nm}$ the size of the halo is dominant $(h \gg \sigma)$, whereas above $6 \mathrm{keV} / \mathrm{nm}$ its contribution becomes negligible, in accordance with a well-defined Poisson behavior for the amorphization kinetics. This reduction of the halo for the higher values of $S_{e}$ is somewhat counterintuitive and could be related to the annealing of the defective zone produced by the high temperatures reached in these cases. A more detailed discussion of these data would require a physical model for the structure of the irradiation-induced tracks.

\footnotetext{
${ }^{1}$ For interpretation of color in Figs. 1-6, the reader is referred to the web version of this article.
}

\section{Physical implications on the structure and mechanisms of SHI damage}

The good agreement between the track-overlap formulae and the experimental amorphization kinetics provides strong support for the cumulative models of track formation, irrespective of the precise microscopic mechanisms. Most theoretical analyses of the amorphization cross-section are based on the thermal spike approach $[17,19]$. However, the significant contribution of the halo in the near-threshold region strongly suggests that one should invoke cumulative models that are consistent with the dual core/ halo morphology of the tracks. In particular, one may use the theoretical scheme developed for $\mathrm{LiNbO}_{3}$, which relies on a strong synergy between the non-radiative exciton decay and the high temperatures reached at the thermal spike. Every ion impact generates a certain radial profile of defect concentration with a nanometric width around the ion trajectory; a Gaussian distribution might be a good option. In fact, the shape of such distribution has to be related to the number of successive impacts $N$ that are required in the overlapping model to induce amorphization. Then, when this distribution reaches a critical local concentration, the crystal lattice collapses through a defect-assisted phase transition and amorphization is induced. The central amorphous (collapsed) region constitutes the core of the track, whereas the surrounding cloud of defects constitutes the so-called defective halo. It can be readily understood that the relative extension of the halo in comparison to the core decreases on increasing stopping power. When two impacts are close enough the two associated defect distributions overlap and the defect concentrations are added, so contributing to the extension of the amorphous area. The situation for the damage kinetics in the electronic regime does not differ much from the one that has been studied for the elastic collision regime (see Section 6). One may, then, understand that the role of the halo is particularly relevant for low stopping powers and decreases for high stopping powers. The data in Fig. 5 indicate that, for stopping powers close to the threshold, the halo is much more extended than the core, as expected. However, no halo is obtained for the higher values of $S_{e}$, showing that it basically plays no role in these cases (i.e., they follow a Poisson kinetics). A more thorough analysis of the data would require a definite physical model yielding the radial profile of the generated defect concentration, as proposed for $\mathrm{LiNbO}_{3}$. For quartz, available data $[46,47]$ point to an electronic mechanism as responsible for the damage. In fact, a number of experimental and theoretical works support [48-51] the non-radiative exciton relaxation as the mechanism accounting for point defect and color center formation. However, a quantitative exciton model to account for the damage features is still pending.

\section{Comparison with amorphization by elastic collisions}

It appears useful at this stage to present a brief comparative discussion of our results with those already achieved for ion-beam damage caused by elastic collisions, which has been more thoroughly investigated. Most information on this type of damage has been collected for low energy $(<100 \mathrm{keV})$ irradiation, where elastic collision processes are clearly dominant. A relevant example is silicon, due to the extensive use of ion-implantation doping in the semiconductor industry [52]. In a simplistic approach it is assumed that amorphization is produced when a critical defect concentration is exceeded. Although this idea contains part of the truth, the situation appears much more complicated as described in several reviews [9-11]. In principle one may distinguish homogeneous mechanisms in which defects do not keep memory of the displacement cascade so that the process of defect accumulation is purely additive and amorphization occurs at a critical (thresh- 
old) defect concentration. However, most models rely on heterogeneous mechanisms, as reported for $\mathrm{ZrSiO}_{4}$ and $\mathrm{SrTiO}_{3}$. They include direct-impact (in-cascade) mechanisms or defect accumulation by cascade overlapping, and nucleation (and growth) limited models. Some combinations of those primary mechanisms are also possible and other effects such as local heating associated to displacement cascades can also play a role in achieving the amorphous phase. Those mechanisms yield quite different kinetic responses [10]: direct processes yield a Poisson-type law whereas for the other mechanisms sigmoidal curves are obeyed.

Our results for the case of electronic excitation damage stress indicate that, in spite of the very different microscopic mechanisms, the kinetic behavior follows similar rules to those for damage by elastic collisions. One has direct amorphization processes by single impacts, that obey approximately a Poisson kinetics (as a result of track overlap) and processes not causing direct amorphization but requiring accumulation of defects coming from different ion impacts. However, there are a number of differences. One of them has to do with the spatial (transversal) scale of the damaged regions (tracks), which are of nanometric size for electronic processes and micrometer size for elastic collisions. On the other hand, the thresholding behavior separating the Poisson and sigmoidal kinetic laws is much sharply defined for electronic damage. Above a well-defined threshold value of the electronic stopping power the behavior can be described by the Poisson kinetics regardless of the type of ion. Below such threshold one has a sigmoidal (Avramilike) kinetics. Our study indicates that the different behavior can be well understood if one takes into account the detailed morphology of the damage track, consisting of an amorphous core and a defective halo. Depending on the relative size of such halo one observes either Poisson (negligible halo) or sigmoidal (relevant halo) behaviors.

\section{Conclusions}

The analysis of the amorphization kinetics for quartz in the electronic stopping regime clearly shows that it can be very well fitted with a cumulative model, assuming that the track is composed of an amorphous core and a surrounding defective halo. The obtained fits are better than those achieved for Avrami-type laws, particularly for electronic stopping powers close to the threshold value. The analysis (that uses the whole kinetic curves and not just the initial slopes) confirms the occurrence of a threshold stopping power at around $2.1 \mathrm{keV} / \mathrm{nm}$. A second fastgrowing zone is observed around $5 \mathrm{keV} / \mathrm{nm}$, revealing a new threshold near $4.1 \mathrm{keV} / \mathrm{nm}$; we believe that this effect could be related with the appearance of discontinuous tracks in the region between both thresholds. Our results indicate that ion irradiation in the electronic regime involves the generation of point defects along the ion trajectory and suggests that this thresholding is associated to the existence of a critical concentration triggering a lattice collapse. Future works should try to assess the microscopic mechanisms, very likely related to non-radiative exciton annihilation in accordance with recent theoretical and experimental work.

\section{Acknowledgements}

This work has been supported by Spanish Ministry MICINN through the project MAT-2008-06794-C03-03, and by Madrid Community through the project TECHNOFUSION (S2009/ENE1679). OPR is grateful to CONACyT, Mexico, for extending a postdoctoral fellowship.

\section{References}

[1] P.D. Townsend, P.J. Chandler, L. Zhang, Optical Effects of Ion Implantation, Cambridge University Press, Cambridge, UK, 1994.

[2] M. Nastasi, J. Mayer, J.K. Hirvonen, Ion-Solid Interactions: Fundamentals and Applications, Cambridge University Press, 1996.

[3] F. Agulló-López, C.R.A. Catlow, P.D. Townsend, Point Defects in Materials, Academic Press, San Diego, CA, USA, 1988.

[4] W.J. Weber, R.C. Ewing, C.R.A. Catlow, T.D. de la Rubia, L.W. Hobbs, C. Kinoshita, et al., J. Mater. Res. 13 (1998) 1434-1484.

[5] I. Farnan, H. Cho, W.J. Weber, Nature 445 (2007) 190-193.

[6] E.R. Hodgson, J. Nucl. Mater. 258-263 (1998) 226-233.

[7] C.D. Marshall, J.A. Speth, L.D. DeLoach, S.A. Payne, Penetrating radiation impact on NIF final optic components, in: Solid State Lasers for Application to Inertial Confinement Fusion Second Annual International Conference, SPIE, 1997, pp. 343-363.

[8] C.W. White, L.A. Boatner, P.S. Sklad, C.J. McHargue, J. Rankin, G.C. Farlow, et al., Nucl. Instrum. Meth. B 32 (1988) 11-22.

[9] A. Meldrum, S.J. Zinkle, L.A. Boatner, R.C. Ewing, Phys. Rev. B 59 (1999) 39813992.

[10] W.J. Weber, Nucl. Instrum. Meth. B 166-167 (2000) 98-106.

[11] A. Meldrum, L.A. Boatner, W.J. Weber, R.C. Ewing, J. Nucl. Mater. $300(2002)$ 242-254.

[12] A. Rivera, J. Olivares, G. García, J.M. Cabrera, F. Agulló-Rueda, F. Agulló-López, Phys. Stat. Sol. A. 206 (2009) 1109-1116

[13] N. Itoh, M. Stoneham, Materials Modification by Electronic Excitation, Cambridge University Press, 2000.

[14] R.L. Fleischer, P.B. Price, R.M. Walker, Nuclear Tracks in Solids: Principles and Applications, University of California Press, Berkeley, 1975.

[15] M. Toulemonde, F. Studer, Solid State Phenom. 30-31 (1992) 477-488.

[16] S. Klaumünzer, Nucl. Instrum. Meth. B 225 (2004) 136-153.

[17] M. Toulemonde, C. Dufour, E. Paumier, Phys. Rev. B 46 (1992) 14362-14369.

[18] A. Meftah, F. Brisard, J.M. Costantini, E. Dooryhee, M. Hage-Ali, M. Hervieu, et al., Phys. Rev. B 49 (1994) 12457-12463.

[19] G. Szenes, Phys. Rev. B 60 (1999) 3140-3147.

[20] F. Agullô-López, G. García, J. Olivares, J. Appl. Phys. 97 (2005) 093514.

[21] F. Agulló-López, A. Mendez, G. García, J. Olivares, J.M. Cabrera, Phys. Rev. B 74 (2006) 174109.

[22] A. Dunlop, D. Lesueur, Rad. Eff. Def. Solids. 126 (1993) 123-128.

[23] G. Sattonnay, S. Moll, L. Thomé, C. Decorse, C. Legros, P. Simon, et al., J. Appl Phys. 108 (2010) 103512-103512-13.

[24] M. Lang, J. Lian, J. Zhang, F. Zhang, W.J. Weber, C. Trautmann, et al., Phys. Rev. B 79 (2009) 224105

[25] G. Sattonnay, C. Grygiel, I. Monnet, C. Legros, M. Herbst-Ghysel, L. Thomé, Acta Mater. 60 (2012) 22-34.

[26] P. Kluth, C.S. Schnohr, O.H. Pakarinen, F. Djurabekova, D.J. Sprouster, R. Giulian, et al., Phys. Rev. Lett. 101 (2008) 175503.

[27] M. Toulemonde, S.M.M. Ramos, H. Bernas, C. Clerc, B. Canut, ]. Chaumont, et al., Nucl. Instrum. Meth. B 178 (2001) 331-336.

[28] G.G. Bentini, M. Bianconi, L. Correra, M. Chiarini, P. Mazzoldi, C. Sada, et al., J. Appl. Phys. 96 (2004) 242-247.

[29] A. García-Navarro, F. Agulló-López, M. Bianconi, J. Olivares, G. García, J. Appl. Phys. 101 (2007) 083506.

[30] M. Bianconi, G.G. Bentini, M. Chiarini, P. De Nicola, G.B. Montanari, A. Nubile, et al., Nucl. Instrum. Meth. B 267 (2009) 2839-2845.

[31] A. Kamarou, W. Wesch, E. Wendler, A. Undisz, M. Rettenmayr, Phys. Rev. B 73 (2006) 184107.

[32] G. García, A. Rivera, M.L. Crespillo, N. Gordillo, J. Olivares, F. Agulló-López, Nucl. Instrum. Meth. B 269 (2011) 492-497.

[33] A. Rivera, M.L. Crespillo, J. Olivares, G. García, F. Agulló-López, Nucl. Instrum. Meth. B 268 (2010) 2249-2256.

[34] O. Peña-Rodríguez, J. Manzano-Santamaría, J. Olivares, A. Rivera, F. AgullóLópez, Nucl. Instrum. Meth. B 277 (2012) 126-130.

[35] J.F. Gibbons, Proc. IEEE. 60 (1972) 1062-1096.

[36] CMAM - Centre for Micro Analysis of Materials: http://www.cmam.uam.es/. (2011).

[37] J. Manzano-Santamaría, J. Olivares, A. Rivera, F. Agulló-López, Nucl. Instrum. Meth. B 272 (2012) 271-274.

[38] J. Ziegler, SRIM - The Stopping and Range of Ions in Matter: http:// www.srim.org/, (2008).

[39] J.F. Ziegler, The Stopping and Range of Ions in Solids, Pergamon Pr, 1985.

[40] L.C. Feldman, J.W. Mayer, S.T.A. Picraux, Materials Analysis by lon Channeling Academic Press, New York, 1982.

[41] At first glance it may seem strange that we get the same value of $N$ for different combinations of ions and substrates. However, this result can be better understood if we remember that in our model $N$ is defined as the number of halos that must overlap to amorphize a particular region. This implies that in the halo region we are somewhat below the damage threshold for the given ion/material combination (because the material would be damaged by the first impact if we were over the threshold and would remain unaffected if we were too far below) and it is, therefore, natural that the same (or similar) number of impacts is needed to cause amorphization.

[42] G. Szenes, Phys. Rev. B 51 (1995) 8026-8029.

[43] A. Rivera, J. Olivares, M.L. Crespillo, G. García, M. Bianconi, F. Agulló-López, Nucl. Instrum. Meth. B 267 (2009) 1460-1463. 
[44] P.l. Gaiduk, A.N. Larsen, C. Trautmann, M. Toulemonde, Phys. Rev. B 66 (2002) 045316.

[45] S.J. Zinkle, V.A. Skuratov, Nucl. Instrum. Meth. B 141 (1998) 737-746.

[46] K. Awazu, S. Ishii, K. Shima, S. Roorda, J.L. Brebner, Phys. Rev. B 62 (2000) 3689-3698

[47] M. Ma, X. Chen, K. Yang, X. Yang, Y. Sun, Y. Jin, et al., Nucl. Instrum. Meth. B 268 (2010) 67-72.
[48] C. Itoh, T. Suzuki, N. Itoh, Phys. Rev. B 41 (1990) 3794-3799.

[49] R.M. Van Ginhoven, H. Jónsson, L.R. Corrales, J. Non-Cryst. Sol. 352 (2006) $2589-2595$.

[50] S. Ismail-Beigi, S.G. Louie, Phys. Rev. Lett. 95 (2005) 156401.

[51] F. Messina, L. Vaccaro, M. Cannas, Phys. Rev. B 81 (2010) 035212.

[52] H. Ryssel, I. Ruge, Ion Implantation, John Wiley \& Sons Inc., New York, NY, 1986. 